\title{
Improving Peer Connectivity in Wide-area Overlays of Virtual Workstations
}

\author{
Arijit Ganguly, P. Oscar Boykin, David I. Wolinksky, Renato J. Figueiredo \\ Advanced Computing and Information Systems Laboratory \\ University of Florida, Gainesville, FL 32611 \\ \{aganguly, boykin, davidiw, renato\}@acis.ufl.edu
}

\begin{abstract}
Self-configuring virtual networks rely on structured P2P routing to provide seamless connectivity among nodes through overlay routing of virtual IP packets, support decentralized hole-punching to establish bi-directional communication links among nodes behind network address translators, and dynamic configuration of virtual IP addresses. Our experiences with deployments of virtual networks in support of wide-area overlays of virtual workstations (WOWs) reveal that connectivity constraints imposed by symmetric NATs and by Internet route outages often hinder P2P overlay structure maintenance and routability, subsequently limiting the ability of WOWs to deliver high-throughput computing through aggregation of resources in different domains.

In this paper, we describe and evaluate two novel approaches which are generally applicable and fully decentralized, and show that they improve routability of structured P2P networks in such connectivity constrained environments: (1) a fault-tolerant routing algorithm based on simulated annealing from optimization theory, and (2) tunneling of connections between adjacent nodes (in the P2P identifier space) over common neighbors when direct communication is not possible. Simulation-based analyses show that when pairs of nodes only have $70 \%$ chance of being able to communicate directly, the described approaches improve all-to-all routability of the network from $90 \%$ to $99 \%$. We have implemented these techniques in the IP-over-P2P (IPOP) virtual network and have conducted experiments with a 180-node WOW Condor pool, demonstrating that, at $81 \%$ probability of establishing a pair-wise connection, annealing and tunneling combined allow all nodes to be connected to the pool, compared to only 160 nodes in the absence of these techniques.
\end{abstract}

\section{Categories and Subject Descriptors}

C.2.4 [Distributed systems]

Permission to make digital or hard copies of all or part of this work for personal or classroom use is granted without fee provided that copies are not made or distributed for profit or commercial advantage and that copies bear this notice and the full citation on the first page. To copy otherwise, to republish, to post on servers or to redistribute to lists, requires prior specific permission and/or a fee.

HPDC'08, June 23-27, 2008, Boston, Massachusetts, USA.

Copyright 2008 ACM 978-1-59593-997-5/08/06 ...\$5.00.

\section{General Terms}

Algorithms, Design, Experimentation

\section{Keywords}

P2P, DHT, virtual network, overlay

\section{INTRODUCTION}

Wide-area overlays of virtual workstations (WOWs) are appealing infrastructures for the creation of high-throughput computing pools and cross-domain collaborative environments ([12][3][23][5]) due to their ability of self-configuring functionally homogeneous virtual networks of virtual machines on top of a heterogeneous wide-area physical infrastructure ([15][32]). Like several related efforts (such as Chord [31], Kademlia [25] and Pastry [30]), WOWs rely on structured $\mathrm{P} 2 \mathrm{P}$ overlays to provide the core service of message routing and additional capabilities such as object storage and retrieval. In the case of WOWs, a structured P2P virtual network (IPOP [14]) provides all-to-all connectivity among nodes, automatic configuration of virtual IP addresses of nodes using a decentralized Dynamic Host Configuration Protocol (DHCP) [16] implementation, and selfoptimization through creation of direct overlay links between virtual IP nodes [15].

Structured P2P routing assumes each node has a consistent view of its local neighborhood in the P2P identifier space, which is reflected in its ability to communicate with its neighboring nodes. However, in practice, widearea environments are becoming increasingly constrained in terms of peer connectivity, primarily due to the proliferation of NAT and firewall routers. These constraints can render structured P2P routing inconsistent, negatively affecting routability and services built upon the assumption of consistent routing. In this paper, we describe and evaluate novel techniques that achieve consistent routing in connectivityconstrained environments and demonstrate their applicability in representative WOW-based high-throughput computing environments.

Studies have shown that about $30 \%-40 \%$ [21] of the nodes in a P2P system are behind NATs. Certain kinds of NAT devices can be "traversed" to support bi-directional communication links through UDP-hole punching, a technique which has been implemented in systems such as IPOP and is known to work for a large variety of "cone" NATs. However, certain scenarios often arise where hole-punching is not possible, such as in "symmetric" NATs. In addition, studies have also shown the existence of permanent or transient 
route outages between pairs of nodes on the Internet; for example, [13] reports $5.2 \%$ pair-wise outages among nodes on PlanetLab [9], and an experiment described in this paper reveals 9 broken structured connections in a 420 -node $\mathrm{P} 2 \mathrm{P}$ ring overlaid on PlanetLab. Together, these connectivity constraints pose a challenge to overlay structure maintenance: two adjacent nodes cannot communicate directly, creating false perceptions of a neighbor not being available. In general, these missing links on a $\mathrm{P} 2 \mathrm{P}$ structure lead to inconsistent routing decisions, and subsequently hinder the correctness of structured routing. As applied to WOWs, they hinder the ability of IPOP to provide all-to-all connectivity among nodes that form a virtual cluster.

Related work has addressed challenges including construction of efficient overlay topologies [18], correct routing of object lookups under churn [28][6], and proximity-aware routing [7]. However, an implicit underlying assumption common in previous work is of an environment where P2P nodes are able to establish direct connections to one another. Deployments of these systems have recognized the problem of overlay structure maintenance when only a small fraction of pairs (about 4\% [20]) cannot communicate with each other [13] [17]. However, from our practical experience with WOW deployments, we have observed that this fraction can be significantly larger due to nodes behind (multiple) NATs, and NATs that are symmetric or do not support "hairpin" translation that preclude hole-punching. To illustrate the negative impact of pair-wise connectivity constraints, results from a simulation-based analysis show that the all-toall routability of a 1000-node ring structured overlay using a conventional (greedy) structured routing algorithm is less than $90 \%$ when there is a $70 \%$ chance that $\mathrm{P} 2 \mathrm{P}$ nodes will be able to communicate directly using TCP or UDP transports.

This paper makes the following contributions. We describe and evaluate two novel, synergistic techniques for fault-tolerant routing and structured overlay maintenance in the presence of network outages: annealing routing, an algorithm based on simulated annealing from optimization theory, and tunnel edges, a technique to establish connections between P2P nodes over common neighbors. These techniques are fully decentralized, self-configuring and generally applicable to any structured P2P system. An implementation of these techniques in IPOP has been demonstrated to operate in actual wide-area PlanetLab deployments as well as in NATed environments with emulated pair-wise outages. The effectiveness of these approaches are analyzed for various system configurations with the aid of analytical models, simulation, and data collected from realistic system deployments. We demonstrate these techniques significantly improve all-to-all routability (with respect to conventional greedy routing) from $90 \%$ to over $99 \%$ at $70 \%$ probability of successful pair-wise connections. We also report on results from realistic NAT-constrained environments through experiments which demonstrate the benefit of annealing routing and tunnel edges to improve connectivity within nodes of a 180-node WOW Condor [22] pool, increasing the fraction of reported worker nodes from $88 \%$ to $100 \%$ at $81 \%$ probability of successful pair-wise connections.

The rest of the paper is organized as follows. In Section 2, we overview several sources of connectivity constraints found in wide-area environments for deployment of desktop grids and collaborative environments. In Section 3 we qualitatively describe the impact of incorrect routing on the func- tioning of WOW distributed systems. We then describe a fault-tolerant annealing routing algorithm that can route messages to their correct destinations, even in the presence of missing overlay links (Section 4.1). In Section 4.2, we describe a technique that allows tunneling of overlay links over connections to other nodes and the implementation of this technique in IPOP. In Section 5, we quantify the improvement in structured routing using these two techniques through simulations. We describe our implementation of tunnel edges in the IPOP system in Section 6. In Section 7, we describe experiments that demonstrate the operation of IPOP in connectivity constrained environments. We discuss related work in Section 8, and conclude the paper in Section 9 .

\section{CONNECTIVITY HAZARDS IN WIDE-AREA NETWORKS}

Several structured P2P systems have been deployed on wide-area infrastructures when participating hosts are on the public Internet. For example, OpenDHT [29] relies on non-firewalled PlanetLab P2P nodes to deploy its DHT; however, nodes behind NATs and firewalls can only act as OpenDHT clients and do not store keys. In order to aggregate the increasing number of hosts behind NATs/firewalls as WOW nodes, the IPOP virtual network must be able to deal with a complex wide-area environment as the one depicted in Figure 1, where typical end users of a P2P system are constrained by NATs in which they do not have the control (or expertise) necessary to set up and maintain firewall exceptions and mappings necessary for NAT traversal.

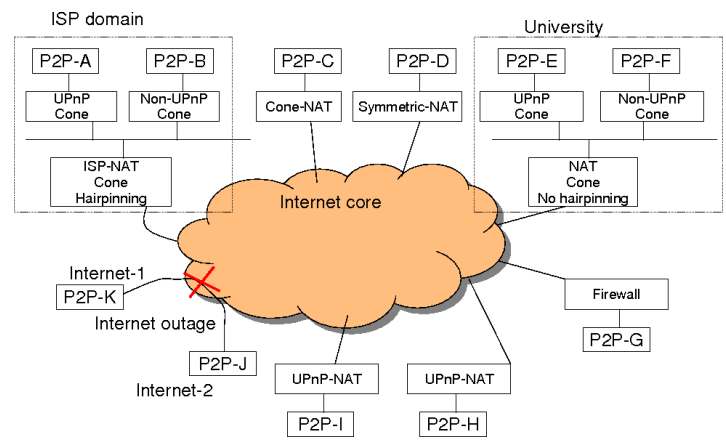

Figure 1: Illustration of the connectivityconstrained wide-area deployment scenario targeted by deployments of the IPOP P2P system.

The scenario which we address in this paper allows the vast majority of $\mathrm{P} 2 \mathrm{P}$ nodes to run on hosts that connect to the Internet through one or more levels of NATs. For example, it is common for broad-band hosts to be behind two levels of NAT (a home gateway/router and the ISP edge NAT, nodes A and B in Figure 1). IPOP supports establishment of UDP communication using hole-punching techniques for "cone" type NATs (e.g. between nodes A and $\mathrm{C}$ in Figure 1), and there is empirical evidence pointing to the fact that these are the common case [11]. Cone NATs consistently map a private IP endpoint to same extenal IP endpoint, irrespective of the destination. These external IP endpoints can be discovered and subsequently exchanged out-of-band between peers in different private networks for hole-punching. 
However, nodes behind "symmetric" NATs for which holepunching does not prove effective cannot communicate with nodes in different private networks, and only communicate with public nodes or full-cone NATs (e.g. nodes C and D). Symmetric NATs map a private IP endpoint a different external IP endpoint for each destination. Hole-punching for symmetric NATs rely on the ability to predict the external IP endpoint prior to communication with the destination such port prediction techniques have not been very effective.

Recognizing the importance of supporting traversal, some of recent NATs have started supporting Universal Plug and Play (UPnP)[2] which allow them to be configured to open ports so that other hosts (outside the NAT) can initiate communication with hosts behind the NAT (e.g. hosts I and H). However, UPnP is not ubiquitous, and even when it is available, multi-level NATs create the problem that hosts can only configure their local NATs through UPnP, while having no access to control the behavior of the edge NAT. This problem renders the UPnP approach ineffective outside the domain. For example, although hosts A and E in Figure 1 are connected to UPnP NATs, they are also subject to rules from an ISP NAT and a University NAT respectively, which they do not control.

Some NATs support "hairpinning", where two nodes in the same private network and behind the same NAT can communicate using each other's translated IP and port. Such a behavior is useful in a multi-level NAT scenario, where two hosts behind the same public NAT but different semipublic NATs are able to communicate only using their IP address and port assigned by the public NAT. However, not all NATs support hairpinning, creating a situation in which two nodes in the same multi-level NATed domain may not be able to use hole-punching to communicate directly (e.g. nodes E and F) as depicted in Figure 2. Through communication with nodes on the public Internet, nodes $\mathrm{E}$ and $\mathrm{F}$ can only learn their IP endpoints assigned by the public NAT-P. In case NAT-P does not support hairpinning, these learned endpoints cannot be used for communication between nodes E and F. Only $24 \%$ of the NATs tested in [11] support hairpinning.
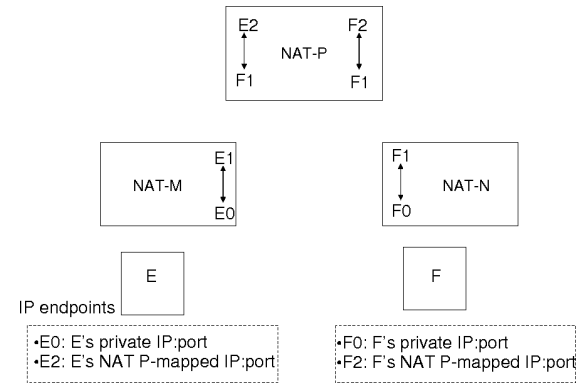

Figure 2: Nodes $E$ and $F$ behind two different semipublic NATs respectively, which in turn are behind a public NAT-P.

Some hosts are behind firewall routers (e.g. host G) that might block all UDP traffic altogether. Only a few P2P nodes are public and are expected to be able to communicate with each other. Connectivity even among these hosts is constrained: Internet-1 and Internet- 2 hosts cannot communicate with each other (e.g. hosts $\mathrm{J}$ and $\mathrm{K}$ ), while multihomed hosts can communicate with them both. In addi- tion, link failures, BGP routing updates, and ISP peering disputes can easily create situations where two public nodes cannot communicate directly with each other. In [13], the authors observed that about $5.2 \%$ of unordered pairs of hosts $(\mathrm{P} 1, \mathrm{P} 2)$ on PlanetLab exhibited a behavior such that $\mathrm{P} 1$ and P2 cannot reach each other but another host P3 can reach both $\mathrm{P} 1$ and P2.

We observe that a typical wide-area environment presents several deterrents to connectivity between a pair of nodes, and when two such nodes have adjacent identifiers on the $\mathrm{P} 2 \mathrm{P}$ ring, structure maintenance is affected. To the best of our knowledge, while structured $\mathrm{P} 2 \mathrm{P}$ systems have been demonstrated in public infrastructures such as PlanetLab, where there are only a few pair-wise outages and a small amount of disorder can be tolerated [17], no structured P2P systems described in the literature have been demonstrated where the majority of P2P nodes are subject to NAT constraints of various kinds as illustrated in Figure 1.

\section{IMPACT OF CONNECTIVITY CONSTRAINTS}

Peer connectivity constraints result in the inability to correctly maintain an overlay structure, which in turn affects the deployment of virtual networks and WOWs in important ways. These are presented and discussed in the remainder of this section.

\subsection{Impact on core structured overlay routing}

The IPOP virtual network implements a ring-structured P2P network where each node has a randomly generated 160-bit identifier. Each node maintains $2 m$ structured near connections, $m$ connections on each side of the $\mathrm{P} 2 \mathrm{P}$ ring. In addition to the neighbor connections, each node also acquires $k$ structured far connections that are far away in the address space, so that the average number of overlay hops between nodes is $O\left(\frac{1}{k} \log ^{2}(n)\right)$ for a network of size $n$ using the algorithm of [19].

Similar to other structured systems, routing in IPOP uses the greedy algorithm where at each hop a message gets monotonically closer to the destination until it is either delivered to the destination or to the node that is closest to the destination in the $\mathrm{P} 2 \mathrm{P}$ identifier space. Greedy routing assumes each node has a consistent view of its local neighborhood, which is reflected in its ability to form structured near connections with its left and right neighbors in the $\mathrm{P} 2 \mathrm{P}$ identifier space. The inability to form connections with immediate neighbors in the identifier space creates inconsistent view of the local neighborhood, thus resulting in incorrect routing decisions as shown in Figure 3 (a). In this figure, nodes 115 and 110 cannot cannot form a connection. A message is sent to key 112, and the closest node is 110 . In one case (a), the message (Create) addressed to key 112 arrives at node 115; it believes that it is the closest to the destination and the message is delivered locally (also replicated at node 100). In another case (b), a message Create addressed to the same key arriving at node 83 is correctly routed to node 110 , the key is not found and created again.

\subsection{Effect on all-to-all connectivity}

Techniques for the creation of overlay links between P2P nodes behind "cone" NATs have been presented in earlier work, which incorporates decentralized NAT traversal using 


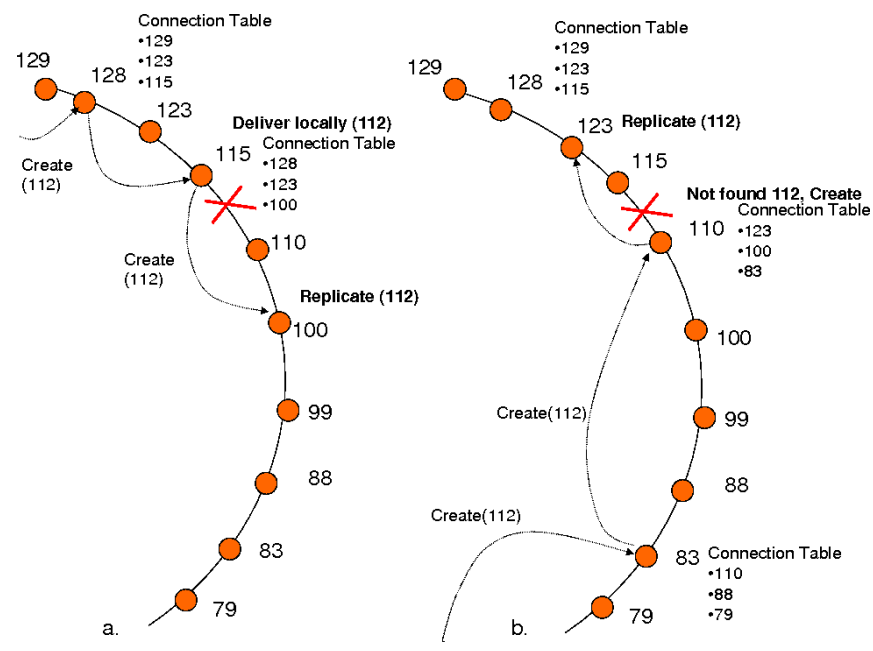

Figure 3: Inconsistent roots in DHT.

UDP hole-punching [15]. The notion of a connection, which describes an overlay link between two P2P nodes, is key to establishing such links. Connections operate over physical channels called edges, which in IPOP can be based on different transports such as UDP or TCP. Besides assisting in overlay structure maintenance, the connection protocols allow the creation of 1-hop shortcuts between WOW nodes to self-optimize the performance of the virtual network with respect to latency and bandwidth.

The connection setup between $\mathrm{P} 2 \mathrm{P}$ nodes is preceded by a connection protocol for conveying the intent to connect and exchanging the list of Uniform Resource Indicators (URIs) for communication. These connection messages are routed over the P2P network. Incorrect routing leads to situations where connection messages are either misdelivered (or not delivered at all), thus affecting both overlay structure maintenance and connectivity within the virtual network.

\subsection{Effect on dynamic virtual IP configuration}

The structured P2P system in IPOP also provides decentralized object storage and retrieval based on a DHT [16], which is used for dynamic virtual IP configuration of WOW nodes, summarized as follows. IPOP supports creation of multiple mutually-isolated virtual networks (called IPOP namespaces) over a common $\mathrm{P} 2 \mathrm{P}$ overlay. The virtual IP configuration of WOW nodes in each such private network is achieved using a decentralized implementation of the Dynamic Host Configuration Protocol (DHCP). The DHCP implementation uses a DHT primitive (called Create) to create key/value pairs mapping virtual network namespaces and virtual IP addresses uniquely to $\mathrm{P} 2 \mathrm{P}$ identifiers. The Create primitive relies on the consistency of key-based routing to guarantee uniqueness of IP-to-P2P address mappings. That is, messages addressed to some key $k$ must be delivered to the same set of nodes regardless of its originator. Incorrect routing decisions can cause Create messages addressed to the same key from different sources to be routed to different nodes, as shown in Figure 3 (a) and (b). This problem is also identified in [13] and is referred to as inconsistent roots, and can lead to a situation where two WOW nodes claim the same virtual IP address.

\subsection{Effect on completion of DHT operations}

To reduce the impact of inconsistent roots, the IPOPDHT internally re-maps each application specified key $k$ to $n$ keys $\left(k_{1}, k_{2} \ldots k_{n}\right)$, which are then stored (together with the associated value) at $n$ different locations on the P2P ring and the DHT operations are expected to separately provide return values for each re-mapped key. Majority voting on results obtained for each such re-mapped key is used to determine the outcome of an operation. For a fault to occur in this scenario, the roots of as many as half of the re-mapped keys have to be inconsistent. However, majority voting can reach a consensus only when results from at least half of the $n$ re-mapped keys are communicated back to the source node. If the nodes close to the source node in identifier space have inconsistent view of their neighborhoods, situations can arise when not enough results arrive at the source node for consensus, causing the operation to fail. This inability to complete a DHT operation impacts both the process of acquiring a virtual IP address, and also resolution of a virtual IP address to P2P identifier.

\subsection{Effect on DHT dynamics}

The inability to create overlay links also hinders the dynamics of a DHT as it reacts to changes in ownership of keys when nodes join, and actively replicates keys when nodes leave. Until a new node can form a consistent view of its local neighborhood by communicating with its neighbors, it can neither retrieve any keys (that it is supposed to store) from its neighbors, nor copy (or migrate) some keys that are now supposed to be stored at its neighbors. This affects the degree of replication of keys in the DHT, and subsequent reliability of object storage.

To summarize, incorrect routing in the P2P network impacts the virtual IP connectivity between WOW nodes, which directly affects the applications using the virtual network. For example, in a WOW-based Condor pool [32], the inability of a worker node to obtain an IP address implies it does not join the pool. Even if a node "N" obtains an IP address, if it cannot communicate with the central manager node "M", it is not available for computation. Furthermore, the inability of node " $\mathrm{N}$ ' to route to a worker node "W" prevents jobs submitted by "N" to execute on "W". All these situations result in the system not being able to achieve the maximum available throughput because not all nodes can participate in computations.

\section{CONSISTENT P2P ROUTING UNDER CONNECTIVITY CONSTRAINTS}

Based on the observations from the previous section, we propose two synergistic approaches to cope with missing overlay links in a structured P2P network: (1) annealing routing and (2) tunnel edges. These are described in the rest of this section.

\subsection{Annealing routing}

The first technique we propose is a fault-tolerant routing algorithm based on simulated annealing that, unlike conventional greedy routing, does not force a message to monotonically get closer to the destination at each hop. This algorithm is inspired by optimization theory. Under the assumption of a convex function, a greedy method converges to a global minimum (or maximum); however, with non-convex 
functions, a greedy approach can stop at local minima and not find the global minimum. In optimization theory, a simulated annealing approach allows for a deviation from greedy search in order to "escape" from a local minimum. In the context of $\mathrm{P} 2 \mathrm{P}$ routing, connectivity constraints create analogous situations where a greedy algorithm can reach a local minimum when a node is not able to establish a near link which would allow the distance between the message and its destination to be reduced. Even if the underlying network is free from connectivity constraints, transient churn can also create situations where a node has an inconsistent view of its local neighborhood. For successful operation of connection setup protocols for overlay structure maintenance, structured routing has to be designed to be tolerant to such disorder on the $\mathrm{P} 2 \mathrm{P}$ ring. The annealing algorithm is described in Algorithm 1 and works as follows.

In lines 10-17, the node looks up its connection table to determine if it is adjacent to the destination in the identifier space. In that case, the node delivers the message locally and also sends it to the node on the other side (left or right) of the destination in the identifier space. ${ }^{1}$ Otherwise (line 19), it finds the two closest nodes to the destination from the connection table, $u_{\min }$ and $u_{\text {sec }}$.

If the message has not taken any hops yet (i.e. it originated at the current node), it is sent to the closest node $u_{\min }$. Otherwise (lines 23-29), until the message has taken MAX_UPHILL hops it is delivered to the closest node $u_{\text {min }}$ or the next closest $u_{\text {sec }}$ (if it was already received from the closest node $\left.u_{\min }\right)$. Until this point, the algorithm does not check for the forward progress of the message towards the destination in identifier space.

Beyond MAX_HILL hops (lines 30-41), the message is sent to $u_{\text {min }}$ or $u_{s e c}$, only if the next hop is closer to the destination than the previous hop. It should be noted that this condition only requires progress with respect to the previous node; it still allows a message take one hop that is farther away from destination than the current node.

The annealing algorithm is very useful for routing messages addressed to exact destinations, which include connection setup messages between $\mathrm{P} 2 \mathrm{P}$ nodes, virtual IP packets between IPOP nodes, and the results of DHT operations back to the source node. In a perfectly-formed structured ring, this algorithm works exactly as the greedy algorithm and incurs the same number of hops.

When messages are addressed to DHT keys, this algorithm has a better chance to reach the node closest to the key, by delivering the message at each local minima. As a side effect, DHT operations for a key are performed at more than one node in the $\mathrm{P} 2 \mathrm{P}$ overlay. This redundancy is useful for applications using only Put and Get DHT interfaces, which do not require uniqueness of key values. However, annealing is not sufficient for scenarios including the decentralized DHCP protocol of IPOP, where it is required to guarantee uniqueness of creation of a key to avoid IP address collisions. Ensuring that each key is delivered to exactly one node (closest to the key in the identifier space) is possible by using greedy routing on a completely formed overlay.

\footnotetext{
${ }^{1}$ The state of the local connection table may not correctly reflect the local neighborhood. While greedy routing may terminate the progress of the message here; by also sending the message to the node on the other side, the annealing algorithm continues its search for the node closest to the destination.
}

Our next technique is designed to provide a complete overlay structure in face of connectivity constraints that may prevent direct connections among overlay neighbors.

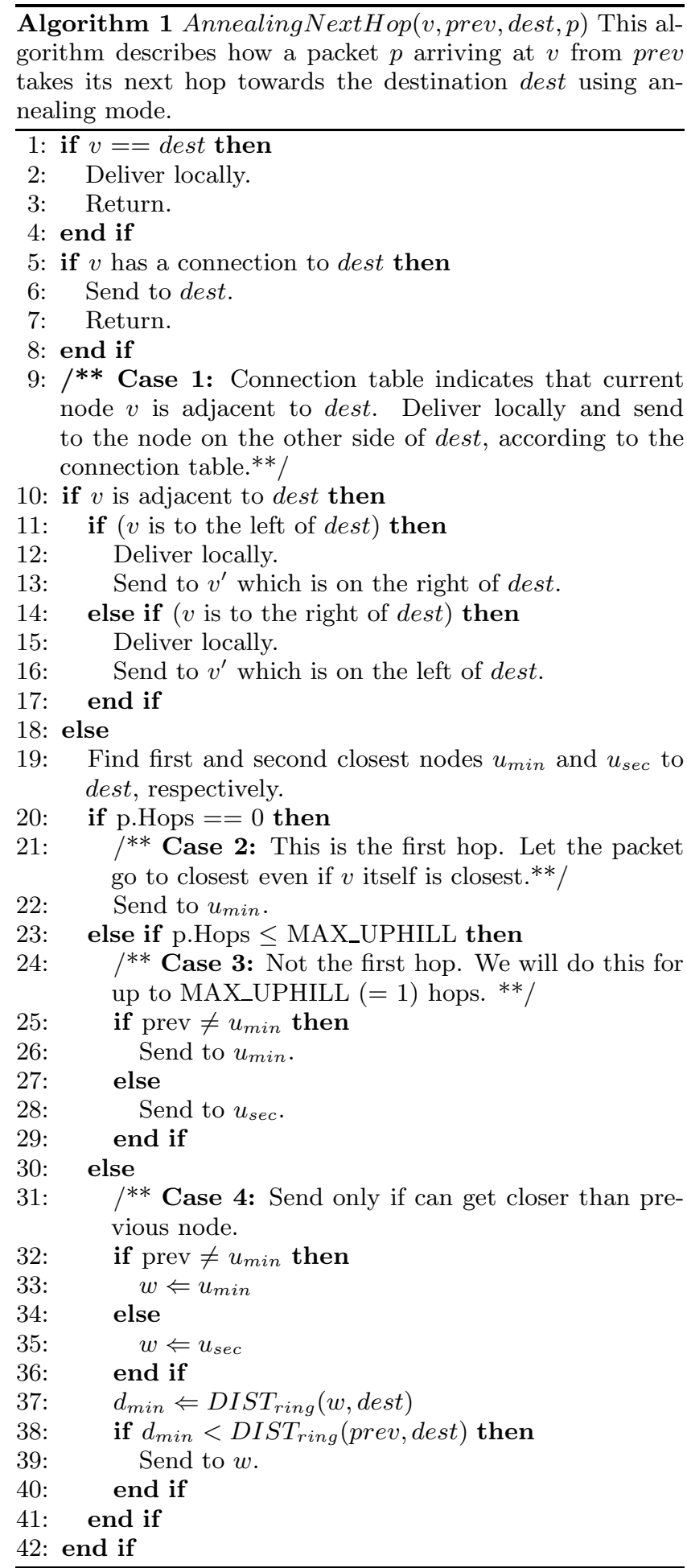

\subsection{Tunnel edges}

In this section, we describe our second novel technique it allows an overlay link between two nodes A and B, which cannot communicate directly over TCP or UDP, to be prox- 
ied by a set nodes to which both $\mathrm{A}$ and $\mathrm{B}$ can communicate. It is a fully decentralized technique for both discovering a proxy node $\mathrm{C}$, and establishing an edge "tunnel" connecting $\mathrm{A}$ and $\mathrm{B}$ through $\mathrm{C}$.

The idea behind tunnel edges is as follows. Assume that each node in the network attempts to acquire connections to its closest $2 m$ near neighbors on the P2P ring, $m$ such neighbors at each side. Consider a situation where there is an outage between two adjacent nodes $\mathrm{A}$ and $\mathrm{B}$ on the $\mathrm{P} 2 \mathrm{P}$ ring. Since both $\mathrm{A}$ and $\mathrm{B}$ also attempt to form near connections with $2 m$ nodes each, their neighborhoods intersect at $2(m-1)$ nodes as shown in Figure 4. For a tunnel edge to exist between $\mathrm{A}$ and $\mathrm{B}$, there must be at least one node $\mathrm{C}$ in the intersection $I$ to which both $\mathrm{A}$ and $\mathrm{B}$ are connected. Such node $\mathrm{C}$ is a candidate to be used in tunneling the structured near connection between $\mathrm{A}$ and $\mathrm{B}$.

This enhancement allows the connection state at a node to consistently reflect the overlay topology even when it is not possible to communicate with some neighbors using the conventional TCP or UDP transports. In the IPOP implementation (described in Section 6), tunnel edges are functionally equivalent to UDP or TCP edges once they are established, allowing seamless reuse of the code responsible for state maintenance and routing logic in the system.

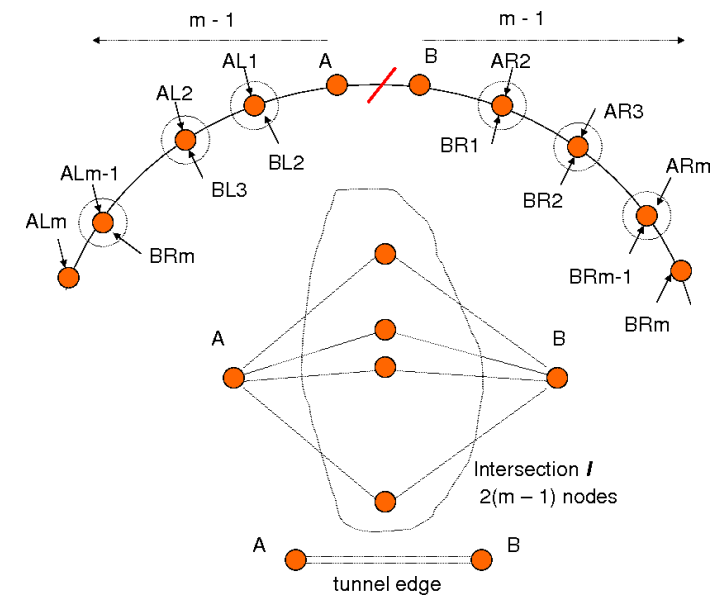

Figure 4: Tunnel edge between nodes $A$ and $B$ that cannot communicate over TCP or UDP transports.

\subsubsection{Probabilistic analysis of tunnel edges}

Two important questions arise in the context of this proposed approach: what is the probability of tunnel edges to be formed between two nodes A and B? how many nodes are candidates for proxying tunnel edges? We address these questions analytically in this section.

Let $q$ be the probability of successful edge setup between a pair of nodes. For a tunnel edge to exist between $\mathrm{A}$ and $\mathrm{B}$, there must be at least one node $\mathrm{C}$ in the intersection $I$ to which both $\mathrm{A}$ and $\mathrm{B}$ are connected. Assuming $m$ near connections at each side of both nodes, the probability for a tunnel edge between $\mathrm{A}$ and $\mathrm{B}$ to exist through $\mathrm{C}$ is given by:

$$
\begin{array}{r}
P[A \text { and } B \text { can connect }] \\
=1-P[A \text { and } B \text { cannot connect }] \\
=1-\prod_{C \in I} P[A \text { and } B \text { cannot connect through } C] \\
=1-\left(1-q^{2}\right)^{2(m-1)}
\end{array}
$$

In Table 1, we show the probability of forming a tunnel edge between unconnected nodes $\mathrm{A}$ and $\mathrm{B}$ for different values of edge probability $q$ and number of near connections $m$. It should be noted that the there is a sharp increase in the probability of being able to form a tunnel edge when nodes acquire more than 2 near connections on each side. This fact is also reflected in simulation results which show that improvements in correctness of routing using tunnel edges are significantly higher when $m \geq 3$. Figure 7 shows $3.9 \%$ broken pairs when $m=2$ and $0.86 \%$ broken pairs when $m=3$.

\begin{tabular}{|l|l|l|l|l|}
\hline & \multicolumn{4}{|c|}{ tunnel edge probability } \\
\hline edge prob & $\mathrm{m}=2$ & $\mathrm{~m}=3$ & $\mathrm{~m}=4$ & $\mathrm{~m}=5$ \\
\hline 0.70 & 0.7399 & 0.9323 & 0.9824 & 0.9954 \\
\hline 0.75 & 0.8085 & 0.9633 & 0.9929 & 0.9986 \\
\hline 0.80 & 0.8704 & 0.9832 & 0.9978 & 0.9997 \\
\hline 0.90 & 0.9638 & 0.9986 & 0.9999 & 0.9999 \\
\hline
\end{tabular}

Table 1: Probability of being able to form a tunnel edge as a function of edge probability and number of required near connections on each side

Now consider a situation where a tunnel edge involves exactly one forwarding node. When the forwarding node departs, the current node also loses the tunnel edge connection. Therefore, for fault-tolerance, it is also important that the forwarding set of nodes for tunnel edge contains more than one node. The probability that that forwarding set consists of at least 2 nodes is given by:

$$
\begin{array}{r}
\quad P[\text { forwarding set of size atleast } 2] \\
=1-\sum_{k=0}^{k=1} P[\text { forwarding set of size exactly } k] \\
=1-\sum_{k=0}^{k=1}\left(\begin{array}{c}
2(m-1) \\
k
\end{array}\right) \cdot\left(q^{2}\right)^{k} \cdot\left(1-q^{2}\right)^{2(m-1)-k}
\end{array}
$$

and the expected size of the forwarding set is given by:

$$
\begin{array}{r}
=\sum_{k=0}^{k=2(m-1)} k \cdot P[\text { forpected size of forwarding set }] \\
=\sum_{k=0}^{k=2(m-1)} k \cdot\left(\begin{array}{c}
2(m-1) \\
k
\end{array}\right) \cdot\left(q^{2}\right)^{k} \cdot\left(1-q^{2}\right)^{2(m-1)-k} \\
=2(m-1) \cdot q^{2}
\end{array}
$$

For $m=3$, and $q=0.9$, the expected size of the forwarding set for a tunnel edge is 3.24 , while the probability of having a forwarding set of at least 2 nodes is 0.976 .

It can further be shown that if each node maintains $O\left(\log _{2} N\right)$ neighbors, then the probability of not being able to form a 
tunnel edge is:

$$
\begin{aligned}
=\left(1-q^{2}\right)^{2(m-1)}=\left(1-q^{2}\right)^{O(m)} & =\left(1-q^{2}\right)^{O\left(\log _{2} N\right)} \\
& =O\left(N^{\log _{2}\left(1-q^{2}\right)}\right)
\end{aligned}
$$

For $q=0.9$, the above expression evaluates to: $O\left(N^{-2.39}\right)$. Therefore, as the network grows in size and nodes tend to acquire more near connections, tunnel edges become more and more probable.

\section{IMPROVEMENTS IN STRUCTURED ROUTING}

In this section, we quantify the improvements in structured routing due to annealing routing and tunnel edges with respect to: (1) the all-to-all routability of the P2P network, and (2) consistent routing of keys. The analysis is conducted by simulating structured routing on randomly generated static graphs that model the IPOP overlay, for varying edge probabilities between pairs of nodes.

Scenarios such as symmetric NATs, multi-level NATs and Internet route outages result in complex models for the likelihood of two nodes being able to communicate. For example, the likelihood of a node behind a symmetric NAT being able to form an edge with another arbitrary node depends on the fraction of nodes that are public (or behind full-cone NATs). In the multi-level NAT scenario (Figure 2) where the outermost NAT-P does not support "hairpinning", the likelihood of a node $\mathrm{E}$ to form an edge with another arbitrary node is a function of the fraction of nodes that are behind the same NAT-P, but in a different semi-private network. An Internet route outage between two sites $\mathrm{A}$ and $\mathrm{B}$ results in the inability of any node in A to communicate with any node in B.

In the absence of any published work that provides a fault model to capture all such scenarios, we model the likelihood of an edge between a pair of nodes with a uniform pairwise edge probability and allow for high probabilities of $\mathrm{P} 2 \mathrm{P}$ edges not being able to form — as high as $30 \%$.

\subsection{Simulation methodology}

The simulation environment captures the algorithms used in IPOP for structured overlay creation and routing, and models pair-wise outages with configurable probability $q$. We create 1000 nodes with randomly generated 160-bit identifiers. Based on the probability $q$ of any pair of nodes being able to communicate using TCP or UDP, we create a connection matrix that allows/disallows connections between pairs of nodes. We then add connections to nodes in the following steps:

1. At each node, attempt to add near connections to the immediate $m$ neighbors (on each side) respecting the connection matrix.

2. If tunneling is enabled: identify all the missing connections between pairs of nodes, compute the overlap of their connection tables to see if tunneling is possible, and add the possible tunnel edges to the network.

3 . If there are nodes with fewer than $m$ connections on each side: each such node tries to acquire more near connections (to its closest neighbors, and fully respecting the connection matrix), until it has successfully acquired $m$ near connections on each side.
4. If there are nodes which acquired more than $m$ connections on each side, these excess connections are trimmed in the subsequent step.

5. We then add one far connection at each node (that is allowed by the connection matrix). The distances traveled by these connections in the structured ring follow the distribution described in [19].

To study the all-to-all routability of the network, we simulate the sending of a message between each pair of nodes, and count the number of times the message is incorrectly delivered. We conduct this experiment for 200 different randomly generated graphs. To investigate correct routing of keys, we randomly generate 10000 different keys. For each key, we simulate the sending of a message addressed to that key from each node as the source, and count the number of times the lookup is wrongly delivered, i.e. to nodes other than the the node closest to the key in identifier space. We conduct this experiment for 200 different randomly generated graphs.

Figure 5 shows the number of non-routable pairs (out of $1000 \times 1000$ possible pairs) of nodes for different values of the number of near neighbors $m$, when neither annealing routing nor tunnel edges are used. We observe that as edge likelihood drops to $70 \%$, the all-to-all routability of the network drops to less than $90 \%$, i.e. more than $10 \%$ of pairs are non-routable. Furthermore, keeping more near connections at each node only marginally improves the network routability. Similar observations are also made for routing of keys (see Figure 6) - there is more than 10\% chance that a key is wrongly routed as the edge likelihood drops to $70 \%$,

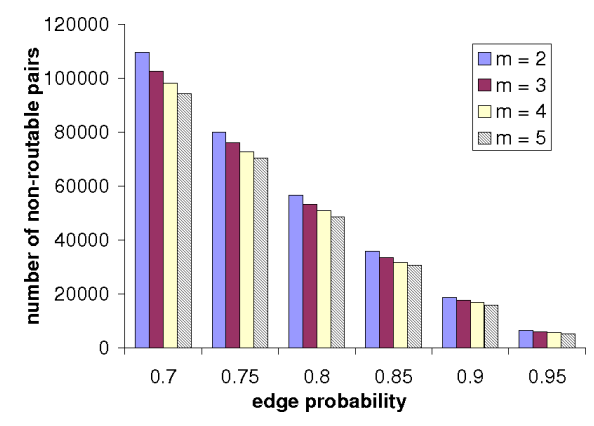

Figure 5: At edge likelihood of $70 \%$ (0.7), the percentage of non-routable pairs varies from $9.5 \%$ to $10.9 \%$ (the total number of pairs in the simulated network is $1,000,000)$.

\subsection{Evaluating the impact of annealing routing}

In Figure 8, we show the reduction in average number of non-routable pairs using Algorithm 1 with $m=3$; tunnel edges are not enabled. We observe that, at an edge likelihood of $85 \%$, the percentage of non-routable pairs with annealing routing is about $0.6 \%$, which is less than one-fifth of the percentage when greedy routing $(3.3 \%)$ is used. Even when the edge likelihood drops to $70 \%$, the percentage of non-routable pairs (less than $3.4 \%$ ) is still less than half of that when greedy routing is used (more than 10\%). It should also be noted that annealing routing with $m=3$ is more likely to reach the correct destination than using 


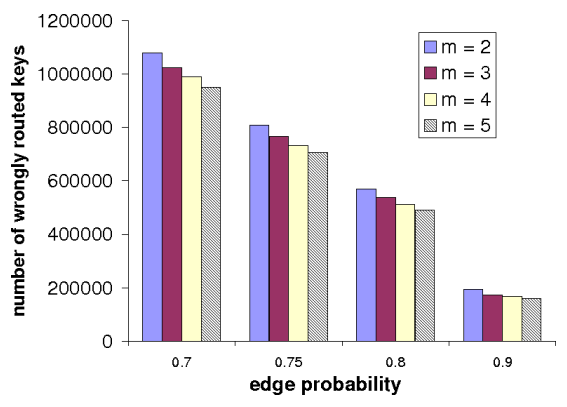

Figure 6: At edge likelihood of $70 \%$, the percentage of wrongly routed keys varies from $9.5 \%$ to $10.7 \%$ (the total number of simulated messages is $10,000,000)$.

greedy routing with $m=5$, for the same edge likelihood in these networks of 1000 nodes.

We measured the average number of hops taken by a message for both greedy and annealing routing for these cases. In a perfectly formed structured network, both routing algorithms incur exactly the same number of hops. Otherwise, the average number of hops between $\mathrm{P} 2 \mathrm{P}$ nodes for annealing is almost the same as for greedy routing. For an edge likelihood of $70 \%$ and $m=3$, the ratio of number of hops incurred by annealing to that of greedy is 1.01. Therefore, annealing routing only incurs a marginal overhead in terms of number of hops.

In Figure 9, we show the average number of wrongly routed key lookups for both annealing and greedy routing using the methodology as described in Section 5.1. We observe at an edge likelihood of $70 \%(m=3)$, annealing routing reduces the chances of a key being wrongly routed from $10.2 \%$ to $3.4 \%$. By delivering a message at more than one node, the annealing algorithm can result in creation of additional (more than two) replicas for a key ${ }^{2}$. The storage overhead due to this additional replication was found to be $9.5 \%$.

\subsection{Evaluating the impact of tunnel edges}

Figure 8 shows how the enhanced overlay structure because of tunnel edges can improve the all-to-all routability of the network, for $m=3$. We observed that even at an edge likelihood of $70 \%$, tunnel edges substantially reduce the percentage of non-routable pairs of nodes from $3.4 \%$ to $0.21 \%$ for annealing routing (from $10 \%$ to $0.86 \%$ for greedy routing).

Each virtual hop over a tunnel edge actually corresponds to two overlay hops. We also recorded the actual number of hops taken by messages addressed to exact destinations in an overlay that supported tunnel edges. For an an edge likelihood of $70 \%$ and $m=3$, the ratio of number of actual hops to that of virtual hops was observed to be 1.14, which is a small overhead considering the improvement in routability.

Figures 9 also compares how tunnel edges improve the consistency of key routability of the network, for $m=3$. We observed that, at an edge likelihood of $70 \%$, with tunnel edges the chances of a key being wrongly routed are $0.86 \%$ for greedy routing (and $0.19 \%$ for annealing routing).

\footnotetext{
${ }^{2}$ Each key in IPOP-DHT is typically replicated at two nodes, on either side of the key in identifier space.
}

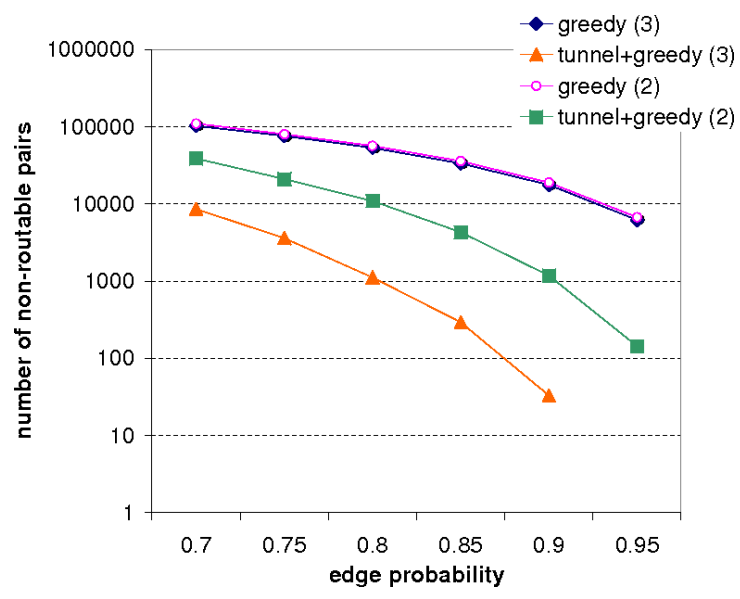

Figure 7: Comparing greedy routing with tunnel edges for $m=3$ and $m=2$. At edge likelihood of $70 \%$, the percentage of non-routable pairs in a network of 1000 nodes is (1) $3.9 \%$ for $m=2$, and (2) $\mathbf{0 . 8 6 \%}$ for $m=3$.

\section{TUNNEL EDGE IMPLEMENTATION IN IPOP}

The IPOP P2P system provides extensive support for creating overlay links between nodes over a variety of transports and incorporates decentralized UDP hole-punching. To implement tunnel edges, we extend the existing mechanisms for connection setup to discover suitable proxy nodes for tunnel edges, when direct communication is not possible.

Connection setup between $\mathrm{P} 2 \mathrm{P}$ nodes is preceded by a connection protocol [15] that uses the $\mathrm{P} 2 \mathrm{P}$ overlay to rendezvous with a remote node for out-of-band exchange of information relevant for communication (through Connect To Me (CTM) messages), followed by a bidirectional linking protocol that establishes the connection. In the original IPOP system, the connection protocol allows nodes to exchange their NAT-assigned IP address/port. In this paper, we use the same mechanism to also exchange information about their connections to near neighbors.

\subsection{TunnelEdgeListener}

As described earlier, each connection in IPOP is based on an edge. Each node has one or more Uniform Resource Indicators (URIs) that abstract the edge protocols it can support and the endpoints over which it can communicate. For each type of edge, an EdgeListener is responsible for creating and maintaining edges of that type, and also sending and receiving messages over connections using that edge type. For example, to create an edge with another node using a URI ipop.udp://128.227.56.123:4000, the UdpEdgeListener is invoked, whereas to communicate with the same node using URI ipop.tcp://128.227.56.123:4001, the TcpEdgeListener is invoked. An IPOP node can have more than one EdgeListener, and new types can be easily added.

Before we further describe the process of creating a tunnel edge, we overview the functionality that allows each IPOP node $\mathrm{C}$ to also act as a message forwarder for communication between two nodes $\mathrm{A}$ and $\mathrm{B}$. The message from the original source $\mathrm{A}$ is encapsulated inside a forward request message addressed to node $\mathrm{C}$. When node $\mathrm{C}$ receives the 


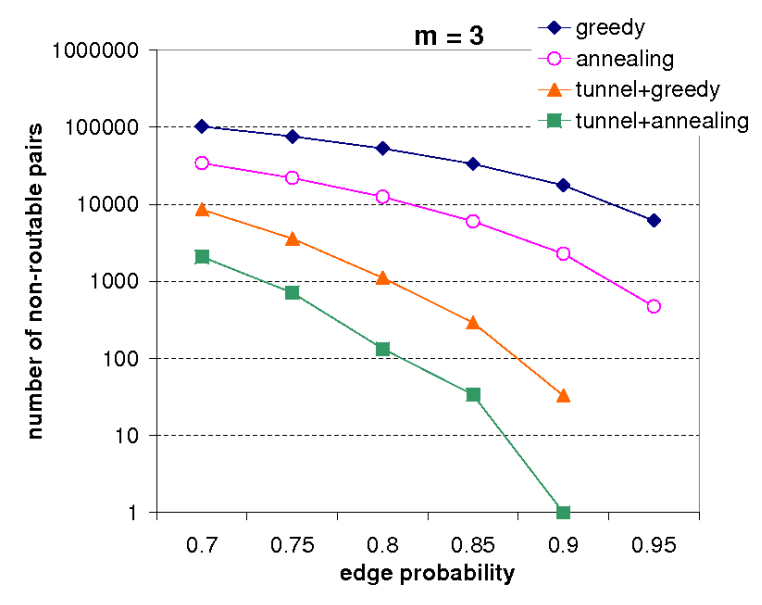

Figure 8: Average number of non-routable pairs. At edge likelihood of $70 \%$, the percentage of nonroutable pairs for greedy and annealing routing is (1) without tunnel edges, $10.26 \%$ and $3.4 \%$ respectively; (2) with tunnel edges, $0.86 \%$ and $0.21 \%$ respectively. At edge likelihoods of $95 \%$, there are no non-routable pairs with tunnel edges.

message from A, it extracts the original message (from A to B), and sends it to node B. This functionality is used by a new IPOP node to identify its left and right neighbors in the $\mathrm{P} 2 \mathrm{P}$ ring [15].

To enable connections between nodes $\mathrm{A}$ and $\mathrm{B}$ to be proxied by common neighbors, we have implemented an EdgeListener called TunnelEdgeListener. The TunnelEdgeListener is invoked together with the TCP and UDP EdgeListeners during the process of a connection setup. The URI for a node corresponding to the tunnel edges is computed dynamically by concatenating the addresses of its closest structured connections. In addition to URIs corresponding to TCP or UDP, nodes also exchange their tunnel URIs inside $C T M$ messages during the connection protocol. Once node A learns about the connections of node B, it can compute the forwarding set $F$ which is the intersection of its own connections with those listed in the tunnel URI of B.

Having computed the forwarding set $F$ with remote node $\mathrm{B}$, the node A sends this information to B in an Edge Request using the forwarding services of one of the nodes in $F$. When $\mathrm{B}$ receives an Edge Request, it replies back with an Edge Response and also records the new tunnel edge. On receiving the Edge Response, the node A also records the new tunnel edge. Once the tunnel edge is successfully created, nodes A and B can subsequently create a connection between them.

Our implementation does not require the nodes in the forwarding set to keep any state about the tunnel edges that are using them. Furthermore, the periodic ping messages to maintain a connection based on a tunnel edge also keep the underlying connections alive. Therefore, no extra overhead is incurred by nodes in the forwarding set. The forwarding set for a tunnel edge can change over time as connections are acquired or lost. To keep the forwarding set up to date and synchronized, nodes A and B notify each other about the changes in their connections.

When a node joins an existing overlay and cannot communicate with its immediate left and right neighbors, its tunnel URI is initially empty since it does not have any connections

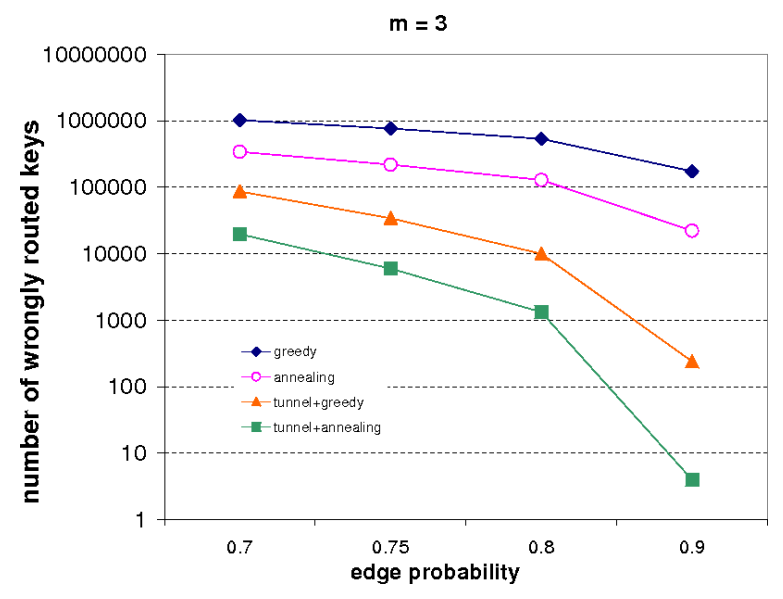

Figure 9: Average number of wrongly routed keys. At edge likelihood of $\mathbf{7 0 \%}$, the percentage of wrongly routed keys for greedy and annealing routing is (1) without tunnel edges, $10.2 \%$ and $3.4 \%$ respectively; (2) with tunnel edges, $0.86 \%$ and $0.19 \%$ respectively.

yet. However, it is possible that the new node can communicate with its other near neighbors, therefore it must first try to form connections with them and then use those connections to form tunnel edges with its immediate neighbors on the P2P ring. The new node learns about its other close neighbors through the $C T M$ messages it receives from its immediate neighbors, which also contain a list of their near connections.

\section{EXPERIMENTS}

In this section, we demonstrate the ability of our tunnel edge implementation to provide a complete ring of $\mathrm{P} 2 \mathrm{P}$ nodes in environments where the majority of nodes are behind NATs and some pairs of nodes cannot communicate with each other directly. We also observe the time it takes for a new node to become connected with its left and right neighbors in an existing $\mathrm{P} 2 \mathrm{P}$ ring, in situations where these connections have to use tunnel edges. Finally, we also study the impact of using annealing routing and tunnel edges on connectivity within a WOW when P2P nodes only had $81 \%$ chance of being able to setup connections.

\subsection{Structure verification of P2P network}

To verify the completeness of the $\mathrm{P} 2 \mathrm{P}$ ring, we iteratively "crawl" the IPOP network using the immediate right neighbor information at each node. We check the consistency of its connections with respect to its predecessor. Specifically, for every node, we check if its immediate left near connection node identifies it as immediate right right connection node.

When two adjacent P2P neighbors cannot form a connection, it is likely that crawling the network using neighbor information will skip a node. If the next reported node has a connection with the missing node, an inconsistency will be reported. However, in case even the second node does not have connection to the missing node, the inconsistency may go unnoticed. It is still possible that we observe a a $100 \%$ consistent ring with a few nodes completely missing. These hidden nodes can be detected using information logged by 
IPOP at each node; knowledge of the number of nodes and their identifiers is also available.

We demonstrate the effect of our techniques with respect to overlay structure, in both a synthetic environment where we artificially create situations where connection setup is not always possible, and also in a large-scale PlanetLab environment, which is known to exhibit route outages between pairs of hosts.

\subsubsection{NATed environment}

To demonstrate the ability of IPOP to deal with heavilyNATed environments, we deployed a P2P network of 1030 nodes involving 12 private networks (each containing $80 \mathrm{P} 2 \mathrm{P}$ nodes) behind port-restricted cone NATs, and a seed network of $70 \mathrm{P} 2 \mathrm{P}$ nodes that was reachable from all other $\mathrm{P} 2 \mathrm{P}$ nodes.

Each node was configured to form connections with 3 neighbors on its immediate left and right. Of the total of 6180 structured near connections reported by all nodes, about 4926 (70\%) existed between nodes which were on different private networks. These connections were not possible without decentralized NAT traversal. The P2P ring was $100 \%$ consistent.

\subsubsection{Incomplete underlying network}

In this experiment, we built a network of $711 \mathrm{P} 2 \mathrm{P}$ nodes incrementally. Starting with a seed network of 71 nodes, we bootstrapped another $640 \mathrm{P} 2 \mathrm{P}$ nodes on 16 hosts (each running $40 \mathrm{P} 2 \mathrm{P}$ nodes). Each $\mathrm{P} 2 \mathrm{P}$ node was configured to use a unique pre-defined UDP port number. Using IPtables, we configured the firewall rules on the hosts to drop UDP packets such that the probability of setting up UDP-based connection between any pair of $\mathrm{P} 2 \mathrm{P}$ nodes was 0.95 .

Once the P2P structure was formed, we observed there were 35 pairs of adjacent nodes on the $\mathrm{P} 2 \mathrm{P}$ ring that could not setup a UDP connection because of firewall rules. These pairs of nodes were however able to connect using tunnel edges, thus rendering a complete $\mathrm{P} 2 \mathrm{P}$ ring.

\subsubsection{Wide-area deployment}

In this experiment, we deployed a network of over 420 nodes on PlanetLab with distinct hosts in North America, South America, Asia, Europe and Australia. We observed that as many as 9 adjacent pairs on P2P ring (in North America, Europe and Asia) could not communicate using TCP or UDP transports. Their inability to connect, which we observed indirectly through the fact that tunnel edges had been created, was verified directly by logging into each host and observing that ICMP messages (and SSH connections) to its peer did not go through either.

To further evaluate the ability of tunnel edges to form, we deployed additional $20 \mathrm{P} 2 \mathrm{P}$ nodes on hosts $\mathrm{H} 1$ and 20 nodes running on host H2. These nodes were configured to use only UDP transports, and their hosts $\mathrm{H} 1$ and $\mathrm{H} 2$ were configured to drop UDP packets between them, thus modeling a scenario where there is a routing outage between two sites. We observed two instances where one of the adjacent pairs was running on $\mathrm{H} 1$, while the other was running on H2. Tunnel edges formed between these nodes in both cases, again rendering a $100 \%$ consistent P2P ring. Without tunnel edges, these nodes would have had inconsistent view of their local neighborhoods (in identifier space), and the messages addressed to them were likely to be misdelivered.
We measure the delay incurred by a new P2P node (on a home desktop) to get connected with its left and right neighbors on the ring using tunnel edges over several trials. The average time to get connected with neighbors is less than 10 seconds, using UDP or TCP. The home desktop did not have an Internet path to a few nodes on PlanetLab and every time it became a neighbor to one of these nodes, it relied on tunnel edges to get connected, which took 41 seconds on average. Our current implementation delays creation of tunnel edges by an arbitrarily chosen interval of 15 seconds (to accommodate for the delay in setting up TCP or UDP connections due to hole-punching or packet losses). However, we also observed cases where it took up to 124 seconds to form tunnel edges with the neighbors. This delay in forming tunnel edges is explained as follows.

The linking protocol for connection setup is executed through one or more linkers; each linker sends link messages using the different URIs of the remote node in parallel until it starts receiving replies. Only one linker is active at a time, during which it sends several link messages over a URI until it starts receiving replies or gives up. Initially, the new node does not have any connections to tunnel over and its tunnel URI is empty. The first linker that is created can thus only succeed using TCP or UDP. When TCP or UDP communication is not possible, it takes several attempts for the linker to finish, and the next linker to be activated. In some cases, the linker containing a usable tunnel URI (created after the node has acquired a few connections) is still waiting in the queue. In future work, we are considering an implementation that would allow updations to the tunnel URI listed in the currently active linker, which would obviate the need to wait for the next linker.

\subsection{Connectivity within a WOW}

In this section, we study the impact of using annealing routing and tunnel edges with respect to improvements in connectivity within a WOW-based Condor pool. Using a bootstrap infrastructure of 20 P2P nodes, we created a WOW consisting of 180 Condor worker nodes and 1 manager. We measure the number of workers reported by the manager (using the condor_status command), which is representative of the achievable throughput of the Condor pool. Furthermore, once a worker has been chosen for job execution by the Condor manager through matchmaking, the process of job submission involves direct communication between the submit node and the worker. We also report on the all-toall connectivity between worker nodes. We send 30 ICMP ping messages from each worker to every other worker, and counted a pair as not being connected if ping reported $100 \%$ packet loss.

Initially P2P edges are allowed to form without constraints. The Condor manager reported all 180 workers, and the workers were all-to-all connected. The P2P ring was $100 \%$ consistent. To create situations where direct communication was not always possible, we configured the UdpEdgeListener at each node to deny UDP-based connections with a probability 0.10 . The probability of two nodes being able to form a UDP-based connection is thus given by: $(1-0.10)^{2}=$ $0.90^{2}=0.81$.

We then configured the $\mathrm{P} 2 \mathrm{P}$ nodes to only use greedy routing and no tunnel edges. The Condor manager reported at most 160 nodes, i.e. only $88 \%$ of the worker nodes were available. In addition, there were 6020 pair-wise worker con- 
nections (out of $180 \times 180$ ) that could not form. In another experiment, we configured the $\mathrm{P} 2 \mathrm{P}$ nodes to use annealing routing but no tunnel edges. The Condor manager reported 177 worker nodes, and there were 859 pairs of workers that could not communicate with each other.

Finally, we configured the P2P nodes to use both annealing routing and tunnel edges. The P2P ring consisting of 201 nodes (20 bootstrap, 1 manager and 180 workers) reported 40 tunnel edges, which formed when UDP communication was denied by one of the UdpEdgeListener between adjacent $\mathrm{P} 2 \mathrm{P}$ nodes. We observed only one inconsistency on the $\mathrm{P} 2 \mathrm{P}$ ring, where a tunnel edge did not form because the P2P nodes did not have any overlap on their UDP-based connections, the overlapping connections were already based on tunnel edges and our existing implementation does not support recursive tunneling. The Condor manager reported all 180 workers, and there were only 7 pairs of workers that could not communicate.

\section{RELATED WORK}

In [24], the authors describe the implementation of a Sockets library that can be used by applications for communication between nodes subject to a variety of constraints in wide-area networks. Our work, on the other hand, investigates an approach where applications can be deployed without any modifications, by providing a virtual network with all-to-all connectivity. Furthermore, our approach is selfconfiguring and fully decentralized.

Structured P2P systems (Chord [31], Pastry [30], Bamboo [1], Kademlia [25]) have primarily focused on efficient overlay topologies [18], reliable routing under churn [28][6], and improving latency of lookups through proximity-aware routing [7]. In [13][17], the authors describe the affect of a few (5\% broken pairs) Internet routing outages on widearea deployments of structured $\mathrm{P} 2 \mathrm{P}$ systems. On the other hand, our focus is to enable overlay structure maintenance when a large majority of nodes are behind NATs, and several scenarios hinder communication between nodes. The technqiues described in this paper faciliate correct structured routing, even when many (up to $30 \%$ ) pairs of nodes cannot communicate directly using TCP or UDP.

In [26], the authors present techniques to provide content/path locality and support for NATs and firewalls, where instances of conventional overlays are configured to form a hierarchy of identifier spaces that reflects administrative boundaries and respects connectivity constraints among networks. In a Grid scenario, however, network constraints are not representative of collaboration boundaries, as virtual organizations (VOs) are known to span across multiple administrative domains.

A technique similar to tunnel edges is also described in [27], in the context of a P2P-based email system built on top of Pastry. Our work, on the other hand, uses tunneling to improve all-to-all virtual-IP connectivity between WOW nodes. We also quantify the impact of the described techniques on structured routing through simulations, under different edge probabilities between nodes. Unmanaged Internet Protocol (UIP) [10] proposes to use tunneling in Kademlia DHT to route between "unmanaged" mobile devices and hosts in ad hoc environments, beyond the hierarchical topologies that make up the current Internet. However, our focus is to facilitate IP communication between Grid resources in different "managed" Internet domains.
In [8], the authors describe an algorithm for providing strong consistency of key-based routing (KBR) in dynamic $\mathrm{P} 2 \mathrm{P}$ environments, characterized by frequent changes in membership due to node arrivals and departures. The improvements in eventual consistency by using the techniques described in this paper can benefit the implementation of the strongly consistent KBR. Similarly, [4] provide asymptotic upper bounds on the number of hops taken by messages under varying rates for link and node failures, and describe heuristics to improve routing under those failures. However, their work does not consider failures of links with neighbor nodes and the subsequent impact on consistent structured routing. To complement fault-tolerant routing, our work also attempts to correct the overlay structure in presence of link failures.

\section{CONCLUSION AND FUTURE WORK}

In this work, we describe and evaluate two synergistic approaches for improving routing in structured P2P networks: annealing routing and tunnel edges. Together, these approaches improve the all-to-all routability of a 1000-node ring structured overlay from $90 \%$ to $99 \%$, when pairs of nodes in the underlying network only have a $70 \%$ chance of being able to communicate. Probabilitic analysis and simulation-based experiments suggest that tunnel edges are effective when each node maintains at least 3 neighbors on each side. Experiments with an implementation demonstrated the ability of IPOP to provide a consistenct P2P ring, even when adjacent pairs of node in identifier space cannot communicate using TCP and UDP, in both synthetic environments and a wide-area deployment. The consistent structured $\mathrm{P} 2 \mathrm{P}$ routing has also been shown to improve the virtual-IP connectivity within a WOW-based condor pool experimentally.

Our current implementation of tunnel edges "passively" relies on an overlap between connections of two nodes, for forming an tunnel edge between them. Symmetric NATed nodes can be difficult to handle using this implementation since they can only communicate with public nodes (or nodes behind cone-NATs). These nodes may not be able to connect with any of their close neighbors. In future work, we plan to change the implementation of tunnel edges to allow nodes to "actively" form connections with common sets of nodes, and then use these connections to form tunnel edges.

\section{REFERENCES}

[1] "the bamboo dht - introduction". http://bamboo-dht.org/.

[2] "universal plug and play in windows xp". http://technet.microsoft.com/enus/library/bb457049.aspx.

[3] D. P. Anderson, J. Cobb, E. Korpella, M. Lebofsky, and D. Werthimer. Seti@home: An experiment in public-resource computing. Communications of the ACM, 11(45):56-61, 2002.

[4] J. Aspnes, Z. Diamadi, and G. Shah. Fault-tolerant routing in peer-to-peer systems. In Proc. of the Symp. on Principles of Distributed Computing (PODC), Monterey, CA, Jul 2002.

[5] B. Calder, A. A. Chien, J. Wang, and D. Yang. The entropia virtual machine for desktop grids. In CSE 
technical report CS2003-0773, University of California, San Diego, San Diego, CA, Oct 2003.

[6] M. Castro, M. Costa, and A. Rowstron. Performance and dependability of structured peer-to-peer overlays. In Proc. of the Conf. on Dependable Systems and Networks, Jun 2004.

[7] M. Castro, P. Druschel, Y. C. Hu, and A. Rowstron. Topology-aware routing in structured peer-to-peer overlay networks. In Microsoft Research MSR-TR-2002-82, Sep 2002.

[8] W. Chen and X. Liu. Enforcing routing consistency in structured peer-to-peer overlays: Should we and could we? In In Proc. of the Workshop on Peer-to-Peer Systems (IPTPS), Santa Barbara, CA, Feb 2006.

[9] B. Chun, D. Culler, T. Roscoe, A. Bavier, L. Peterson, M. Wawrzoniak, and M. Bowman. Planetlab: An overlay testbed for broad-coverage services. $A C M$ SIGCOMM Computer Communication Review, 33(3), 2003.

[10] B. Ford. Unmanaged Internet Protrocol: Taming the edge network management crisis. In Proc. of the Workshop on Hot Topics in Networks (HotNets), Cambridge, MA, Nov 2003.

[11] B. Ford, P. Srisuresh, and D. Kegel. Peer-to-peer communication across network address translators. In Proc. of the USENIX Annual Technical Conference, Anaheim, California, Apr 2005.

[12] I. Foster, C. Kesselman, and S. Tuecke. The anatomy of the grid: Enabling scalable virtual organizations. Intl. Journal of Supercomputer Applications, 15(3), 2001.

[13] M. J. Freedman, K. Lakshminarayanan, S. Rhea, , and I. Stoica. Non-transitive connectivity and DHTs. In Proc. of the USENIX WORLDS, San Francisco, CA, Dec 2005.

[14] A. Ganguly, A. Agrawal, P. O. Boykin, and R. J. Figueiredo. IP over P2P: Enabling self-configuring virtual IP networks for grid computing. In Proc. of Intl. Parallel and Distributed Processing Symp. (IPDPS), Rhodes, Greece, Apr 2006.

[15] A. Ganguly, A. Agrawal, P. O. Boykin, and R. J. Figueiredo. Wow: Self-organizing wide area overlay networks of virtual workstations. In Proc. of Intl. Symp. on High Performance Distributed Computing, Paris, France, Jun 2006.

[16] A. Ganguly, D. Wolinsky, P. O. Boykin, and R. J. Figueiredo. Decentralized dynamic host configuration in wide-area overlays of virtual workstations. In Proc. of the Workshop on Desktop Grids and Volunteer Computing Systems, with IPDPS, Long Beach, CA, Mar 2006.

[17] S. Gerding and J. Stribling. Examining the trade-offs of structrured overlays in dynamic non-transitive network, Dec 2003. Class project: http://pdos.lcs.mit.edu/ strib/doc/networking_fall2003.ps.

[18] K. Gummadi, R. Gummadi, S.Gribble, S. Ratnasamy, S. Shenker, and I. Stoica. The impact of DHT routing geometry on resilience and proximity. In Proc. of ACM SIGCOMM, Karlsruhe, Germany, Aug 2003.
[19] J. Kleinberg. Nature, 406:845, 2000.

[20] J. Li, J. Stribling, R. Morris, M. F. Kaashoek, and T. M. Gil. A performance vs. cost framework for evaluating DHT design tradeoffs under churn. In Proc. IEEE INFOCOM, 2005.

[21] J. Liang, R. Kumar, and K. Ross. The fasttrack overlay: A measurement study. In Computer Networks (Special Issue on Overlays), 2005.

[22] M. Litzkow, M. Livny, and M. Mutka. Condor - a Hunter of Idle Workstations. In Proc. of Intl. Conference on Distributed Computing Systems, Jun 1988.

[23] V. Lo, D. Zappala, D. Zhou, Y. Liu, and S. Zhao. Cluster computing on the fly: P2P scheduling of idle cycles in the internet. In Proc. of the 3rd Intl. Workshop on Peer-to-Peer Systems (IPTPS), San Diego, CA, Feb 2004.

[24] J. Maassen and H. E. Bal. Smartsockets: Solving the connectivity problems in grid computing. In Proc. of Symp. on High Performance Distributed Computing Symposium, Monterey Bay, CA, Jun 2007.

[25] P. Maymounkov and D. Mazières. Kademlia: A peer-to-peer information system based on the xor metric. In Proc. of the Workshop on Peer-to-Peer Systems (IPTPS), Cambridge, MA, Mar 2002.

[26] A. Mislove and P. Druschel. Providing administrative control and autonomy in structured peer-to-peer overlays. In Proc. of the Workshop on Peer-to-peer systems, San Diego, CA, Feb 2004.

[27] A. Mislove, A. Post, A. Haeberlen, and P. Druschel. Experiences in building and operating epost, a reliable peer-to-peer application. In Proc. of European Conf. on Computer Systems, Leuven, Belgium, Apr 2006.

[28] S. Rhea, D. Geels, T. Roscoe, and J. Kubiatowicz. Handling churn in a DHT. In Proc. of USENIX Technical Conference, Jun 2004.

[29] S. Rhea, B. Godfrey, B. Karp, J. Kubiatowicz, S. Ratnasamy, S. Shenker, I. Stoica, and H. Yu. Opendht: A public DHT service and its uses. In Proc. of ACM SIGCOMM, Philadelphia, PA, Aug 2005.

[30] A. Rowstron and P. Druschel. Pastry: Scalable, decentralized object location and routing for large-scale peer-to-peer systems. In Proc. of the Intl. Conf. on Distributed Systems Platforms (Middleware), Heidelberg, Germany, Nov 2001.

[31] I. Stoica, R. Morris, D. Liben-Nowell, D. R. Karger, M. F. Kaashoek, F. Dabek, and H. Balakrishnan. Chord: a scalable peer-to-peer lookup protocol for internet applications. IEEE/ACM Transactions on Networking, 11(1):17-32, 2003.

[32] D. Wolinsky, A. Agrawal, P. O. Boykin, J. Davis, A. Ganguly, V. Paramygin, P. Sheng, and R. Figueiredo. On the design of virtual machine sandboxes for distributed computing in wide area overlays of virtual workstations. In Proc. of the Workshop on Virtualization Technology in Distributed Computing, with Supercomputing, Tampa, FL, Nov 2006. 